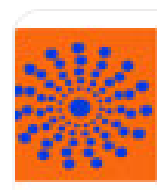

JOURNAL OF BEHA VIOR, HEAtTH de SOCIAL issur

ints

Serrano Vargas, Mario Amado; Montes Castro, Edgar Eduardo EFECTOS DE LAS RELACIONES DE CONDICIONALIDAD EN TAREAS DE IGUALACIÓN DE LA MUESTRA

Journal of Behavior, Health \& Social Issues, vol. 6, núm. 1, mayo-octubre, 2014, pp. 67-78 Asociación Mexicana de Comportamiento y Salud, A. C.

Distrito Federal, México 


\title{
EFECTOS DE LAS RELACIONES DE CONDICIONALIDAD EN TAREAS DE IGUALACION DE LA MUESTRA
}

\author{
EFFECTS OF CONDITIONALITY RELATIONS IN MATCHING-TO- \\ SAMPLE TASKS
}

Mario Amado Serrano Vargas.

Universidad Veracruzana- CEICAH, Xalapa, Veracruz, México.

Edgar Eduardo Montes Castro.

Universidad Enrique Díaz de León, Guadalajara, Jalisco, México.

\author{
Recibido: Junio 25, 2013 \\ Revisado: Noviembre 16, 2013 \\ Aceptado: Diciembre 16, 1013
}

\begin{abstract}
El presente estudio se vio apoyado parcialmente por el proyecto CONACYT 180619 otorgado al primer autor. Edgar Montes fue apoyado por la misma institución mediante la Beca \#177165 para realizar estudios de doctorado. La correspondencia relacionada deberá enviarse a Mario Serrano a: Universidad Veracruzana, Centro de Estudios e Investigaciones en Conocimiento y Aprendizaje Humano. Av. Orizaba No. 203. Fraccionamiento Veracruz. Xalapa, Veracruz. México, C. P. 91020 o bien al correo electrónico mserranoduv.mx
\end{abstract}

El presente estudio evaluó si la transferencia del comportamiento depende de la complejidad funcional de la tarea inicial. Cuatro grupos de estudiantes universitarios fueron expuestos a tareas de igualación de la muestra y dos tipos de ensayos de transferencia: intramodal/extrarelacional y extramodal/extrarelacional. Entre grupos, las tareas difirieron tanto en las relaciones de condicionalidad (i.e., discriminación condicional versus discriminación simple) como en los requisitos de respuesta (i.e., instrumental versus observacional). Los porcentajes de respuestas correctas más elevados en las pruebas de transferencia se observaron para el grupo expuesto a la situación de discriminación condicional y el requisito de respuesta instrumental. Los resultados se discuten en relación con estudios previos sobre los efectos de contingencias intrasituacionales sobre el comportamiento lingüísticamente regulado y la variabilidad.

Palabras clave: Discriminación simple, discriminación condicional, entrenamiento observacional, igualación de la muestra, transferencia, humanos. 


\section{Abstract}

The present study assessed if the transfer of behavior depends on the functional complexity of the initial task. Four groups of college students were exposed to second-order matching-to-samples tasks and two kinds of transfer trials: intramodal/extrarelational and extramodal/extrarelational. Between groups, tasks differed in both conditionality relations (i.e., conditional discrimination versus simple discrimination) and response requirements (i.e., instrumental versus observational). The highest percentage of correct responses in transfer tests were observed for the group exposed to the conditional discrimination situation and the instrumental response requirement. Results are discussed in relation to previous experiments on the effects of intrasituational contingencies upon linguistically-regulated behavior and variability.

Key words: Simple discrimination, conditional discrimination, observational training, matching-tosample tasks, transfer, humans.

\section{Introducción}

La llamada función selectora de la propuesta taxonómica de Ribes y López (1985), describe una interacción psicológica caracterizada porque la funcionalidad de las propiedades fisicoquímicas de los objetos y eventos con los que el organismo interactúa varía momento a momento. Una misma característica fisicoquímica puede tener más de una propiedad funcional al interior de un arreglo de contingencias, o bien diversas características fisicoquímicas pueden compartir una misma propiedad funcional. En la medida que implica la independización del responder respecto de las características fisicoquímicas de los eventos de estímulo, se estima que la configuración del comportamiento al nivel de la función selectora constituye una condición crítica para transitar hacia formas de comportamiento reguladas lingüísticamente, es decir, interacciones cuyas relaciones de condicionalidad no dependen de las propiedades dimensionales de los eventos involucrados sino de formas sociales de interacción ante ellas (Ribes, 2012; Ribes, Ibáñez \& Hernández-Pozo, 1986).

La configuración del comportamiento al nivel de la función selectora y su posible tránsito hacia formas de comportamiento lingüísticamente reguladas se ha estudiado principalmente mediante tareas de igualación de la muestra de primer y segundo ordenes (Moreno, Cepeda, Tena, Hickman \& Plancarte, 2005; Peña-Correal, Ordoñez, Fonseca, \& Fonseca, 2012; Ribes, 1998; Tena, Hickman, Moreno, Cepeda \& Larios, 2001). En ambas tareas se presentan un estímulo de muestra y dos o más estímulos de comparación, de entre los cuales un participante debe elegir aquel que guarda con el estímulo de muestra una relación de igualación preestablecida. Las tareas se distinguen entre sí porque en la tarea de igualación de la muestra de segundo orden se presentan, adicionalmente, dos estímulos que instruyen visualmente la relación de igualación a satisfacer en cada ensayo. En ambas tareas, la regulación lingüística del comportamiento se infiere a partir de la ejecución exitosa en pruebas de transferencia en las que la retroalimentación se cancela y se introducen nuevas relaciones de igualación, o bien se introducen cambios en la dimensión o ámbito de desempeño en el que las tareas tuvieron lugar durante el entrenamiento (véase Ribes, Vargas, Luna \& Martínez, 2009; Varela \& Quintana, 1995).

Las relaciones de igualación en las tareas de igualación de la muestra de primer y segundo ordenes pueden establecerse con base en cualquiera de las propiedades fisicoquímicas de las instancias de estímulo, sin embargo, el carácter funcionalmente relevante e irrelevante de tales propiedades debe variar entre los ensayos de igualación. Si la funcionalidad de las propiedades fisicoquímicas pertinentes de igualación no permuta entre ensayos, las contingencias de ocurrencia (Ribes, 1997) no corresponderían con aquellas que posibilitan la configuración de la función selectora sino formas de comportamiento menos complejas. 
Si la funcionalidad de las propiedades fisicoquímicas de los eventos de estímulo permanece constante y el contacto entre tales eventos está determinado por la actividad del organismo, las contingencias de ocurrencia involucradas corresponden con aquellas que auspician la configuración del comportamiento al nivel de la Ilamada función suplementaria. Si la funcionalidad de las propiedades fisicoquímicas de los eventos de estímulo es constante o variable, pero el contacto entre tales eventos es inalterable por parte del organismo, entonces las contingencias de ocurrencia involucradas corresponden con aquellas que auspician la configuración del comportamiento al nivel de la Ilamada función contextual (Carpio et al., 2001).

Si la configuración del comportamiento al nivel de la función selectora constituye una condición crítica para transitar hacia formas de comportamiento lingüísticamente reguladas, es probable que la configuración del comportamiento bajo contingencias de ocurrencia que auspician funciones menos complejas limite dicho tránsito. Dicho en otros términos iqué nivel de complejidad funcional deben implicar las contingencias a partir de las que tiene lugar el tránsito hacia formas de comportamiento reguladas lingüísticamente? El presente experimento trató de responder esta pregunta, comparando los efectos de tareas de igualación de la muestra de segundo orden que implicaban discriminaciones simples o condicionales, bajo condiciones observacionales o instrumentales, sobre la precisión de la ejecución en ensayos de transferencia.

\section{Método}

\section{Participantes}

En el estudio participaron 12 estudiantes de segundo semestre de la Facultad de Psicología de la Universidad Veracruzana (UV), Campus Xalapa. Los participantes eran experimentalmente ingenuos en tareas de igualación de la muestra y sus edades fluctuaron entre los 18 y 23 años. Los participantes fueron divididos al azar en cuatro grupos de tres sujetos cada uno: (a) Selector; (b) Suplementario; (c) Contextual variable; y (d) Contextual constante.

\section{Aparatos}

El estudio se llevó a cabo en tres de los cubículos de experimentación del Laboratorio Móvil de Aprendizaje Humano Sidney Bijou del Centro de Estudios e Investigaciones en Conocimiento y Aprendizaje Humano de la UV. Además de repisa y silla, cada uno de los cubículos contó con una computadora personal estándar (HP Compaq Modelo, dc5850) provista con monitor, teclado y mouse. Las instrucciones e instancias de estímulo de las tareas experimentales fueron elaboradas en mapas de bites independientes y organizadas mediante el programa SuperLab Pro ${ }^{\circledR}$ (Versión 2.0) en un ambiente Windows ${ }^{\circledR}$. El mouse funcionó como dispositivo para las respuestas de igualación, las cuales se registraron automáticamente por las computadoras. Las sesiones experimentales se realizaron entre las 18:00 y las 20:00 horas.

\section{Procedimiento}

Los cuatro grupos de participantes fueron expuestos a una preprueba, una sesión de entrenamiento instrumental u observacional y una prueba de transferencia compuesta por ensayos aleatorios de tipo intramodal, intramodal/extrarelacional, extramodal y extramodal/extrarelacional. Las tres fases del experimento se condujeron en una misma sesión para cada grupo, sin embargo, los grupos Contextual variable y Contextual constante fueron evaluados siete días después de la evaluación a la que fueron sometidos los grupos Selector y Suplementario.

\section{Preprueba}

La preprueba consistió en 54 ensayos de igualación de la muestra de segundo orden en la dimensión geométrica: (a) nueve ensayos de semejanza en color; (b) nueve ensayos de semejanza en forma; (c) nueve ensayos de diferencia en color y forma; (d) nueve ensayos de semejanza en contorno; (e) nueve ensayos de semejanza en tamaño; $y$ (f) nueve ensayos de diferencia en tamaño y forma o bien en contorno y forma. Los ensayos se presentaron de manera aleatoria y se extrajeron al azar de los ensayos del entrenamiento y las pruebas de transferencia. En cada ensayo se presentaron dos estímulos de segundo orden en la parte superior de la pantalla, un estímulo de muestra en la parte 
central y cuatro estímulos de comparación en la parte inferior. Los ensayos basados en el color y la forma de las figuras geométricas se diseñaron de tal forma que siempre existió un estímulo de comparación idéntico, uno semejante en color, otro semejante en forma y uno diferente en color y forma respecto del estímulo de muestra. En los ensayos basados en el contorno y la forma de las figuras geométricas siempre existió un estímulo de comparación idéntico, uno semejante en contorno, otro semejante en forma y uno diferente en forma y contorno respecto del estímulo de muestra. En los ensayos basados en el tamaño y la forma de las figuras geométricas siempre existió un estímulo de comparación idéntico, uno semejante en tamaño, otro semejante en forma y uno diferente en forma y tamaño respecto del estímulo de muestra.

Para todos los grupos las instrucciones de la preprueba fueron:

"En las siguientes pantallas aparecerán siete figuras geométricas: dos en la parte superior, una en el centro y cuatro en la parte inferior de la pantalla. Elige la figura de abajo que corresponda con la del centro de acuerdo con lo que indican las dos figuras de arriba. Para registrar tu respuesta, ubica el puntero del "mouse" dentro de la figura que elegiste y oprime el botón izquierdo. Para iniciar oprime el botón COMENZAR".

\section{Entrenamiento}

El entrenamiento consistió en 96 ensayos de igualación de la muestra de segundo orden en la dimensión geométrica basados en el color y la forma de las figuras. La mitad de los ensayos implicaron la relación de semejanza en color y la otra mitad implicaron la relación de semejanza en forma. Para los participantes de los grupos Selector y Suplementario, los ensayos de entrenamiento se presentaron de manera aleatoria y fueron precedidos por una instrucción general similar a la de la preprueba, excepto porque se agregó el enunciado "Te informaremos si tu respuesta fue correcta o incorrecta". Para ambos grupos de participantes las respuestas de igualación acertadas y erróneas produjeron la desaparición del arreglo de igualación y, respectivamente, la presentación de las palabras correcto e inco- rrecto durante 3 s. Posteriormente se presentó un intervalo entre ensayos de $3 \mathrm{~s}$.

Los participantes de los grupos Contextual variable y Contextual constante se "acoplaron" a los participantes de los grupos Selector y Suplementario, respectivamente. Para los participantes de los dos grupos contextuales las respuestas de igualación no fueron requeridas y cada ensayo estuvo vigente durante $3 \mathrm{~s}$. Para cada participante de los grupos contextuales se enmarcó en un cuadrado rojo alguno de los cuatro estímulos de comparación, según el ensayo presentado y la ejecución registrada para su contraparte en los grupos Selector o Suplementario. Dependiendo de si se trataba de un estímulo de comparación acertado o erróneo, las palabras correcto o incorrecto se presentaron una vez terminado el ensayo. Las instrucciones para los participantes de los grupos Contextual Variable y Contextual Constante fueron:

“En las siguientes pantallas aparecerán siete figuras geométricas: dos en la parte superior, una en el centro y cuatro en la parte inferior de la pantalla. Una de las figuras de abajo se enmarcará mediante un cuadrado rojo y podrá o no corresponder con la figura del centro, de acuerdo con lo que indican las dos figuras de arriba. Te informaremos si la figura enmarcada es correcta o incorrecta. Para iniciar oprime el botón COMENZAR".

A partir de las figuras círculo y cruz y los colores azul y morado se conformaron ocho parejas de estímulos de segundo orden. Cuatro parejas modelaron la relación de semejanza en color y cuatro parejas modelaron la relación de semejanza en forma. Un primer arreglo de primer orden se conformó a partir de un triángulo isósceles verde, un cuadrado verde, un triángulo rojo y un pentágono amarillo, los cuales variaron su posición de forma aleatoria entre los ensayos cuando se presentaron como estímulos de comparación. Un segundo arreglo de primer orden se conformó a partir de un triángulo escaleno lila, un trapecio lila, un triángulo escaleno crema y un octágono negro, los cuales también variaron su posición de forma aleatoria entre los ensayos. Para los participantes de los grupos 
Selector y Contextual variable, ambos arreglos de igualación de la muestra de primer orden se correlacionaron con las 8 parejas de estímulos de segundo orden. Para los participantes de los grupos Suplementario y Contextual constante, el primer arreglo de primer orden se correlacionó diferencialmente con las cuatro parejas de estímulos de segundo orden que modelaron la relación de semejanza en color, mientras el segundo arreglo de primer orden se correlacionó diferencialmente con las cuatro parejas de estímulos de segundo orden que modelaron la relación de semejanza en forma.

La Figura 1 muestra las relaciones de condicionalidad a las que fueron expuestos los participantes de cada grupo. Las flechas punteadas corresponden con las relaciones de condicionalidad a las que fueron expuestos los participantes de los grupos Selector y Contextual variable. Las flechas continuas corresponden a las relaciones de condicionalidad a las que fueron expuestos los participantes de los grupos Suplementario y Contextual constante. Nótese que para los participantes de los dos primeros grupos antes mencionados los estímulos de comparación correctos entre los ensayos de igualación fueron el cuadrado verde, el triángulo rojo, el trapecio lila y el triángulo escaleno crema. Para los grupos Suplementario y Contextual constante los estímulos de comparación correctos entre los ensayos de igualación fueron únicamente el cuadrado verde y el triángulo escaleno crema.

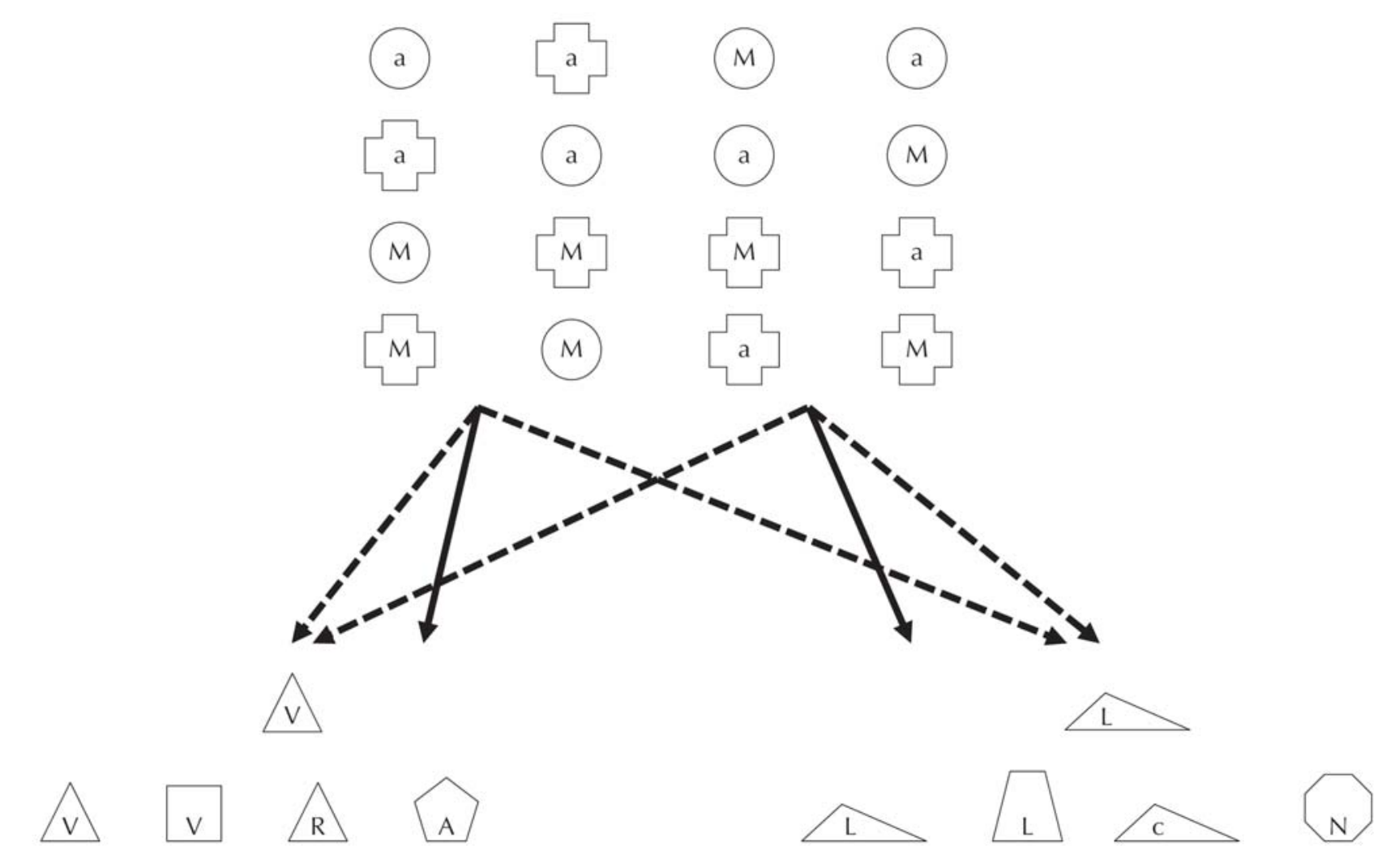

Figura 1. Relaciones de condicionalidad entre los arreglos de segundo y primer orden de las tareas de igualación de la muestra de segundo orden del presente estudio. Las flechas punteadas corresponden con las relaciones de condicionalidad a las que fueron expuestos los participantes de los grupos Selector y Contextual variable, mientras las flechas continuas corresponden a las relaciones de condicionalidad a las que fueron expuestos los participantes de los grupos Suplementario y Contextual constante. ( $\mathrm{a}=$ azul, $\mathrm{M}=$ morado, $\mathrm{V}=$ verde, $\mathrm{R}=$ rojo y $\mathrm{A}=$ amarillo). 
Ensayos de transferencia

Los ensayos implementados en la fase de transferencia fueron similares a los ensayos presentados en la fase de preprueba, excepto porque el número se incrementó a 72 ensayos de igualación de la muestra de segundo orden. La mitad de los ensayos se basó en el color y la forma de las figuras geométricas y la otra mitad se basó en el contorno y el tamaño de las figuras. De los ensayos basados en el color y la forma de las figuras, 12 ensayos fueron de semejanza en color, 12 ensayos fueron de semejanza en forma y 12 ensayos fueron de diferencia en forma y color. De los ensayos basados en el contorno y el tamaño de las figuras, 12 ensayos fueron de semejanza en contorno, 12 ensayos fueron de semejanza en tamaño y 12 ensayos fueron de diferencia. De estos últimos, la mitad de los ensayos fue de diferencia en contorno y forma y la otra mitad fue de diferencia en tamaño y forma.

En los ensayos basados en el color y la forma de las figuras se utilizaron las mismas figuras y colores que en la fase de entrenamiento para conformar las parejas de estímulos de segundo orden. Para el arreglo de primer orden se utilizó un triángulo rectángulo azul rey, un paralelogramo azul rey, un triángulo rectángulo café y un decágono gris, los cuales variaron aleatoriamente su posición entre los ensayos como estímulos de comparación. En los ensayos basados en el contorno o en el tamaño de las figuras también se utilizaron las mismas figuras que en la fase de entrenamiento para conformar las parejas de estímulos de segundo orden. Las figuras se presentaron en color blanco y sus contornos y sus tamaños variaron. En los ensayos basados en el contorno de las figuras, el círculo y la cruz tuvieron un contorno sólido de 6 ó .25 puntos de ancho y el tamaño se mantuvo constante en $1 \mathrm{~cm} \times 1 \mathrm{~cm}$. En los ensayos basados en el tamaño de las figuras, ambos estímulos tuvieron un tamaño de $2 \times 2 \mathrm{~cm}$ o bien de $.5 \mathrm{~cm} \times .5 \mathrm{~cm}$ y el contorno sólido se mantuvo constante en 6 puntos de ancho. Para los arreglos de primer orden se utilizaron el triángulo rectángulo, el paralelogramo y el decágono, los cuales variaron su posición aleatoriamente entre los ensayos de igualación y se presentaron en color blanco. El triángulo rectángulo tuvo un contorno de punto redondo o de guión largo y en ambos casos un tamaño de $2 \mathrm{~cm} \times 2 \mathrm{~cm}$ en los ensayos basados en el contorno de las figuras. En los ensayos basados en el tamaño de las figuras, el triángulo rectángulo tuvo un tamaño de $1 \mathrm{~cm} \times 1 \mathrm{~cm}$ o bien de 2 $\mathrm{cm} \times 2 \mathrm{~cm}$ y en ambos casos un contorno sólido de 2 puntos de ancho. En los ensayos basados en el contorno de las figuras, el paralelogramo tuvo un tamaño de $2 \mathrm{~cm} \times 2 \mathrm{~cm}$ y un contorno de punto redondo de 2 puntos de ancho. En los en los ensayos basados en el tamaño de las figuras, el paralelogramo tuvo un contorno sólido de 2 puntos de ancho y un tamaño de $1 \mathrm{~cm} \times 1 \mathrm{~cm}$. El decágono tuvo un contorno compuesto doble de punto cuadrado y un tamaño de $2 \mathrm{~cm} \times 2 \mathrm{~cm}$ en los ensayos basados en el contorno de las figuras. En los ensayos basados en el tamaño de las figuras, el decágono tuvo un tamaño de $2 \mathrm{~cm}$ x $2 \mathrm{~cm}$ y un contorno compuesto doble sólido de 2 puntos de ancho.

\section{Resultados}

La Tabla 1 muestra el porcentaje de respuestas correctas para los participantes de cada grupo a lo largo del experimento. Para los tres participantes del grupo Selector se observó un 0\% de respuestas correctas en la preprueba. En esa misma fase, para P4 y P6 del grupo Suplementario la ejecución fue menor al $10 \%$ de aciertos, mientras para P5 fue de un 33\% de respuestas correctas. Para los participantes del grupo Contextual variable, la ejecución osciló entre 0 y $17 \%$ de respuestas correctas, mientras para los participantes del grupo Contextual constante se observaron ejecuciones entre el 13 y el $38 \%$ de aciertos. En la fase de entrenamiento, la ejecución osciló entre 77 y 95\% de respuestas correctas para los participantes del grupo Selector, y entre 82 y $93 \%$ de aciertos para los participantes del grupo Suplementario.

En los ensayos de transferencia intramodal/ extrarelacional se observó un $100 \%$ de aciertos para los tres participantes del grupo Selector y $33 \%$ de aciertos para los tres participantes del grupo Suplementario. Una ejecución similar a esta última se observó en esos mismos ensayos para uno de los participantes del grupo Contextual variable y para dos participantes del grupo 
Contextual constante. Para los dos participantes restantes del grupo Contextual variable se observaron ejecuciones de 0 y $67 \%$ de aciertos, mientras para el participante restante del grupo Contextual constante se observó un $8 \%$ de respuestas correctas. En los ensayos de transferencia extramodal/extrarelacional, la ejecución osciló entre 75 y $100 \%$ de respuestas correctas para los participantes del grupo Selector, mientras para los participantes del grupo Suplementario la ejecución osciló entre 0 y $67 \%$ de respuestas correctas. Para P7 y P9 del grupo Contextual variable se observó un $8 \%$ de respuestas correctas en los ensayos de transferencia extramodal/ extrarelacional, mientras para P8 se observó un $42 \%$ de aciertos. Para los participantes del grupo Contextual constante se observaron ejecuciones de 0,25 y $33 \%$ de respuestas correctas.

Tabla 1.

Porcentaje de respuestas en cada fase del experimento para los participantes de cada grupo.

\begin{tabular}{lrrrrr}
\hline & & \multicolumn{3}{c}{ Fase } \\
\cline { 2 - 5 } Grupo & Participante & PP & E & TIMER & TEMER \\
\hline Selector & P1 & 0 & 95 & 100 & 75 \\
& P2 & 0 & 94 & 100 & 100 \\
Suplementario & P3 & 0 & 77 & 100 & 92 \\
& P4 & 4 & 93 & 33 & 0 \\
Contextual Variable & P5 & 33 & 82 & 33 & 25 \\
& P6 & 8 & 89 & 33 & 67 \\
Contextual Constante & P7 & 17 & & 0 & 8 \\
& P8 & 13 & & 67 & 42 \\
& P9 & 0 & & 33 & 8 \\
& P10 & 13 & & 33 & 25 \\
& P11 & 25 & & 33 & 33 \\
\hline
\end{tabular}

Nota. $\mathrm{PP}=$ preprueba, $\mathrm{E}=$ entrenamiento, $\mathrm{TIMER}=$ transferencia intramodal/extrarelacional y TEMER=transferencia extramodal/extrarelacional.

La Figura 2 muestra el porcentaje de respuestas correctas por relación de igualación para los participantes de los cuatro grupos en los ensayos de transferencia intramodal/extrarelacional (barras en escala de grises) y extramodal/extrarelacional (barras blancas y blancas con líneas diagonales). En la figura se observa que únicamente para los participantes del grupo Selector los porcentajes de respuestas correctas en las relaciones de igualación de diferencia oscilaron entre el 75 y el $100 \%$ de aciertos en los ensayos de transferencia tanto intramodal/extrarelacional como extramodal/extrarelacional. Adicionalmente, en la figura se observa que únicamente en el caso del grupo Selector los porcentajes de respuestas correctas en las relaciones de igualación de semejanza en contorno y semejanza en tamaño fueron del $100 \%$ para dos de los tres participantes en los ensayos de transferencia extramodal/ extrarelacional. 
GRUPO SELECTOR

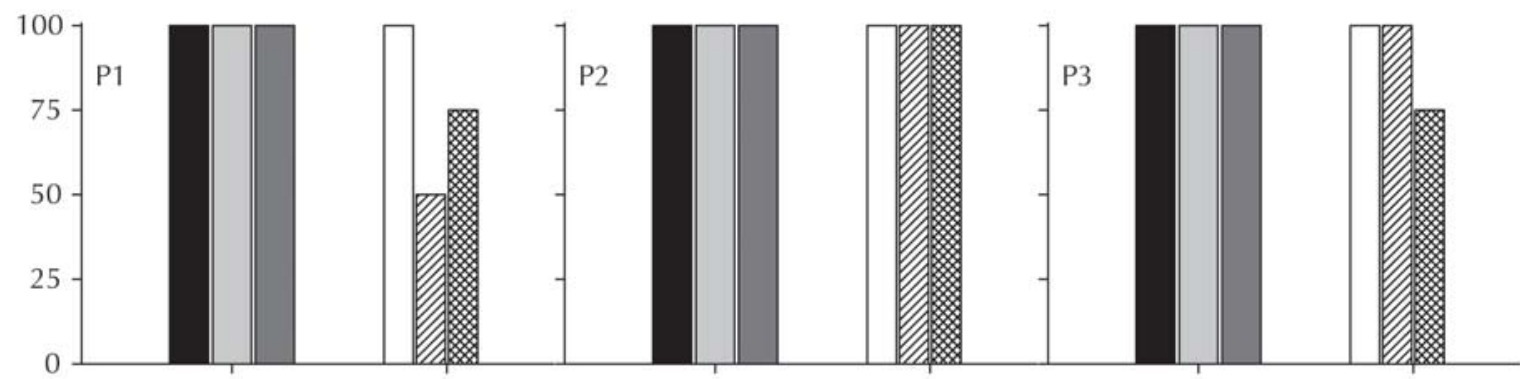

GRUPO SUPLEMENTARIO

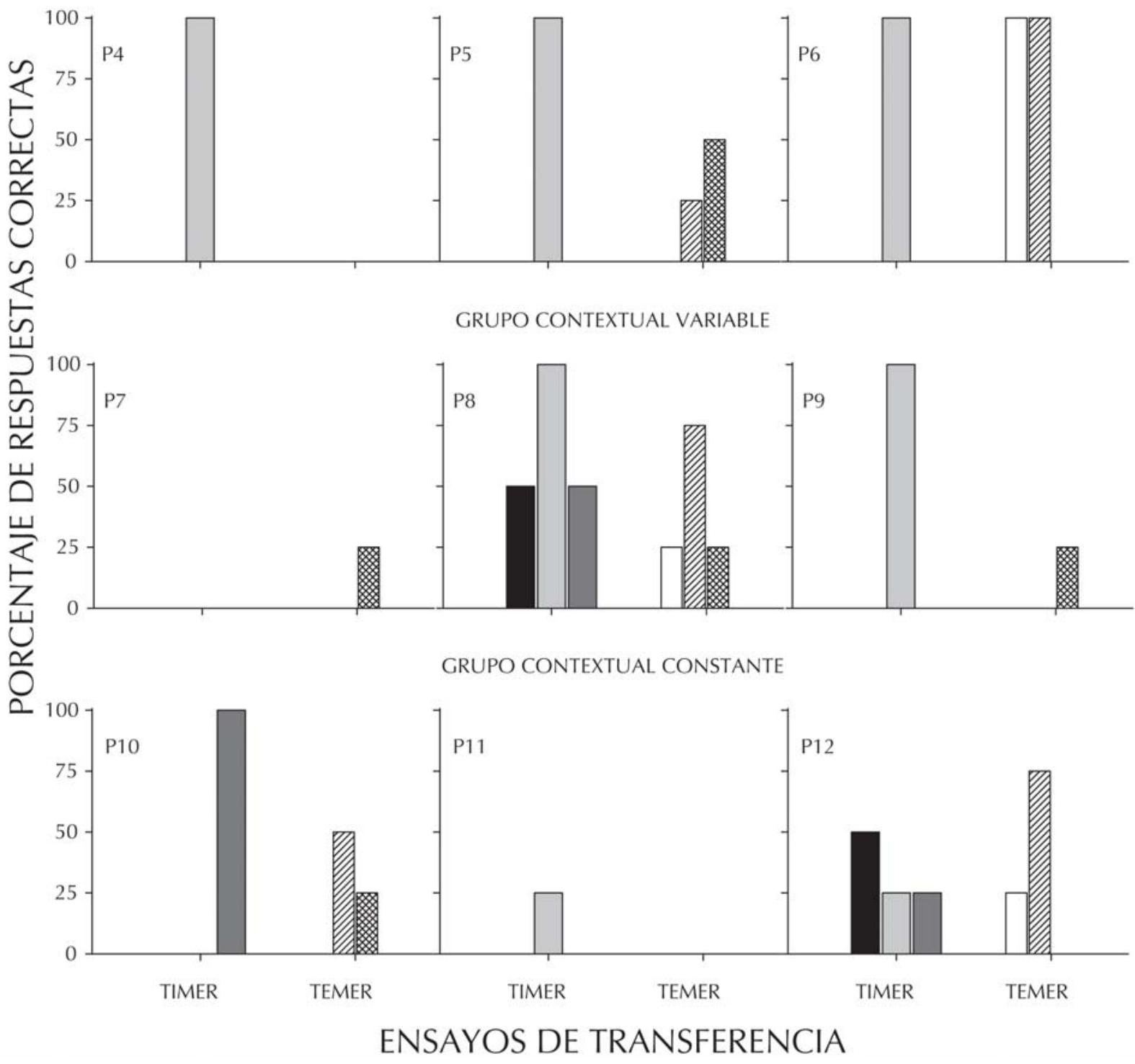

Figura 2. Porcentaje de respuestas correctas por relación de igualación en los ensayos de transferencia intramodal/extrarelacional (TIMER) y extramodal/extrarelacional (TEMER) para los participantes de los cuatro grupos. 
Para los tres participantes del grupo Suplementario se observaron ejecuciones del $0 \%$ de aciertos en las relaciones de diferencia, tanto en los ensayos de transferencia extramodal/extrarelacional como en los ensayos de transferencia intramodal/extrarelacional. En estos últimos, para los participantes del grupo Suplementario los aciertos se concentraron en la relación de semejanza en forma. Únicamente para P6 se observaron ejecuciones del $100 \%$ de aciertos en las relaciones de semejanza de los ensayos de transferencia extramodal/extrarelacional. Para los tres participantes del grupo Contextual variable se observaron ejecuciones del $25 \%$ de respuestas correctas en la relación de igualación de diferencia en los ensayos de transferencia extramodal/extrarelacional, mientras en los ensayos de transferencia intramodal/extrarelacional únicamente para P8 se observó una ejecución superior al $0 \%$ de aciertos. En general, para los participantes de este grupo los aciertos en los ensayos de transferencia se concentraron en las relaciones de semejanza. De los participantes del grupo Contextual constante, sólo para P10 se registró una ejecución superior a cero en una relación de diferencia.

\section{Discusión}

El presente experimento comparó los efectos de tareas de igualación de la muestra de segundo orden que implicaban discriminaciones simples o discriminaciones condicionales, bajo condiciones instrumentales u observacionales, sobre la precisión de la ejecución en ensayos de transferencia. Se observó que la tarea que implicaba discriminaciones condicionales y que requirió de respuestas de igualación en el entrenamiento, produjo las ejecuciones más elevadas en los ensayos de transferencia; tanto en los que implicaron las relaciones entrenadas como en los que implicaron las relaciones no entrenadas. En virtud de las ejecuciones entre 75 y $100 \%$ de aciertos observadas en estas últimas relaciones para los participantes del grupo Selector, los resultados del presente estudio concuerdan con la idea de que la configuración del comportamiento al nivel de la función selectora constituye una condición crítica para transitar hacia formas de comportamiento reguladas lingüísticamente (Ribes, 2012; Ribes et al., 1986; Ribes \& López, 1985).

Dicho en otros términos, lo inmediatamente anterior significa que el tránsito hacia formas de comportamiento lingüísticamente reguladas es más probable mientras mayor sea la complejidad funcional de las contingencias a partir de las cuales dicho tránsito puede tener lugar. Estudios previos han evaluado esta posibilidad con resultados distintos. Ribes et al. (2009), por ejemplo, utilizando diversas versiones de una tarea de igualación de la muestra de segundo orden, expusieron a cinco grupos de participantes a uno, dos, tres, cuatro o cinco tipos de entrenamiento y pruebas de transferencia. Un grupo fue expuesto a cinco tipos de entrenamiento, cada uno implicando la complejidad funcional propia de los cinco niveles de organización del comportamiento reconocidos en la propuesta taxonómica de Ribes y López (1985). Para el segundo grupo se omitió el entrenamiento contextual, para el tercero se omitió tanto el entrenamiento contextual como el entrenamiento suplementario, y así sucesivamente. De acuerdo con dichos autores, haber sido expuesto a contingencias de ocurrencia que posibilitan la evolución de las funciones contextual, suplementaria y/o selectora no favoreció la ejecución en los entrenamientos y pruebas de transferencia que implicaban una regulación necesariamente lingüística del comportamiento. En ambos casos se observaron ejecuciones alrededor del $60 \%$ de aciertos, incluso para los participantes expuestos directamente a tales condiciones.

En un estudio más reciente, Ramírez y PeñaCorreal (2011) entrenaron nuevas morfologías lingüísticas a partir de las ya existentes bajo contingencias de ocurrencia correspondientes a las de las funciones contextual, suplementaria o selectora, y probaron el uso de dichas morfologías en la construcción de oraciones y la identificación de dibujos a los que se referían tales oraciones. El entrenamiento contextual consistió en la presentación de una sílaba sin sentido y medio segundo después un dibujo. En el entrenamiento suplementario, la sílaba sin sentido se presentó con un cuadro de texto en el que los participantes debían escribir el nombre correlacionado. En el entrenamiento 
selector, el nombre del objeto se presentó como estímulo de muestra de una tarea de igualación y tres sílabas sin sentido se presentaron como estímulos de comparación. Después de escoger una sílaba sin sentido, el participante debía escribir la palabra presentada como estímulo de muestra. Se observó que los puntajes tanto de elaboración de oraciones como de identificación de los dibujos correspondientes incrementaron con la complejidad del entrenamiento al que fueron expuestos los participantes.

Dado que en el estudio de Ribes et al. (2009) las ejecuciones en las tareas que implicaban la regulación lingüística del comportamiento fueron bajas incluso para los participantes expuestos directamente a tales condiciones, es posible suponer que la complejidad de tales tareas pudo opacar los efectos de haber sido expuesto a las contingencias de ocurrencia más simples. Los resultados del experimento de Ramírez y Peña-Correal (2011), junto con los descritos en el presente reporte, sugieren que los diseños de grupo son más sensibles que los diseños de línea base múltiple para evaluar los efectos de las contingencias contextuales, suplementarias y selectoras sobre el comportamiento lingüísticamente regulado. Adicionalmente, ambos estudios demuestran la importancia de que los entrenamientos de tipo contextual sean estrictamente observacionales para la identificación de dichos efectos. De hecho, en el caso particular del presente estudio, no incluir a los grupos Contextual variable y Contextual constante hubiera abierto la puerta a una explicación de los resultados observados para los grupos Selector y Suplementario basada en la mera variabilidad y no en las relaciones de condicionalidad implicadas.

Específicamente, estudios previos han demostrado que la variabilidad al interior de las tareas de igualación de la muestra es un factor determinante de la ejecución en las pruebas de transferencia. Cepeda, Moreno y Larios (2000), por ejemplo, expusieron a dos grupos de estudiantes universitarios a tareas de igualación de la muestra de segundo orden y pruebas de transferencia similares a las aquí implementadas. La diferencia entre los grupos radicó en el número de entrenamientos y de relaciones de igualación, así como en el número de figuras y colores que conformaron las instancias de estímulo. En general, observaron un mayor porcentaje de respuestas correctas para los participantes expuestos a las condiciones de mayor variabilidad. En el caso del presente experimento el número de instancias de estímulo se mantuvo constante entre las tareas de igualación de la muestra, sin embargo, implícitamente se auspiciaron grados de variabilidad distintos en términos de los estímulos de comparación funcionalmente relevantes en cada caso. Específicamente, mientras para los participantes de los grupos Suplementario y Contextual constante los estímulos de comparación funcionalmente relevantes fueron únicamente dos, para los participantes de los grupos Selector y Contextual variable existieron cuatro estímulos de comparación funcionalmente relevantes. La omisión de un requisito de respuesta en el caso de los grupos contextuales, sin embargo, apoya la idea de que son las relaciones de condicionalidad propias de la función selectora (i.e., la variación funcional en las propiedades fisicoquímicas de los eventos de estímulo y la respuesta que media suplementariamente la relación contextual entre ellos) y no la mera variabilidad las responsables de las ejecuciones observadas para los participantes del grupo Selector. Debe destacarse, sin embargo, que mientras en el caso del grupo Contextual variable se observó un $25 \%$ de respuestas correctas en las relaciones de diferencia extramodal para los tres participantes, en el caso del grupo Contextual constante sólo para un participante se observó una ejecución similar. Nuevos estudios interesados en abordar el problema evaluado en el presente experimento deberán controlar el número de estímulos de comparación funcionalmente relevantes en las condiciones selectora y suplementaria, así como los efectos de la variabilidad implicada en contingencias contextuales sobre la configuración del comportamiento lingüísticamente regulado.

En general, la conclusión que se desprende de los resultados del presente estudio es que la posibilidad de transitar hacia formas de comportamiento reguladas lingüísticamente es mayor mientras más complejas sean las contingencias a partir de las que dicho tránsito tiene lugar. Antes de finalizar, sin embargo, debe señalarse que el hecho de que el mencionado transito 
haya sido posible sólo a partir de una situación de discriminación condicional con requisito de respuesta, parece revelar una diferencia entre los procesos implicados en la adquisición y transferencia de discriminaciones condicionales tal como se estudian en el ámbito de la psicología interconductual, y la llamada formación de clases de estímulos equivalentes (Sidman \& Tailby, 1982) que se estudia en el ámbito del análisis experimental de la conducta. Específicamente, mientras tales clases pueden formarse bajo condiciones de entrenamiento puramente observacionales (e.g., Delgado-Delgado \& Medina-Arboleda, 2011; Tonneau \& Gonzáles, 2004), la transferencia del comportamiento a nuevas relaciones de igualación sólo parece posible si existen las relaciones de condicionalidad propias de la llamada función selectora (Ribes \& López, 1985). Dicho en otros términos, mientras el responder relacional implicado en las clases de estímulos equivalentes podría explicarse con base en los parámetros del condicionamiento clásico (Pavlov, 1927), la transferencia del responder a nuevas relaciones de igualación requeriría atender a elementos que van más allá de tales parámetros (e. g., el lenguaje). Nuevos experimentos comparativos probablemente revelen las virtudes de una u otra aproximación conceptual en torno del comportamiento lingüísticamente regulado.

\section{Referencias}

Carpio, C., Flores, C., Bautista, E., González, F., Pacheco, V., Páez, A., \& Canales, C. (2001). Análisis experimental de las funciones contextual y selectora. En G. Mares \& Y. Guevara (Eds.), Psicología interconductual: Avances en la investigación básica (pp. 9-35). México: Universidad Nacional Autónoma de México.

Cepeda, M., Moreno, D. \& Larios, R. (2000). Relación de un entrenamiento variado con opciones textuales y la transferencia en una tarea de discriminación condicional. Revista Psicología y Ciencia Social, 2, 3-16.

Delgado-Delgado, D. M., \& Medina-Arboleda I. F. (2011). Efectos de dos tipos de entrenamiento respondiente sobre la formación de relaciones de equivalencia. Revista Mexicana de Análisis de la Conducta, 37, 33-50. doi:10.5514/rmac. v37.i1.19475

Moreno, D., Cepeda, M., Tena, O., Hickman, H., \& Plancarte, P. (2005). Conducta gobernada por reglas: Implicaciones educativas. En C. Carpio \& J. J. Irigoyen (Eds.), Psicología y educación: Aportaciones desde la teoría de la conducta (pp.175-212). México: Universidad Nacional Autónoma de México-Facultad de Estudios Superiores Iztacala.

Pavlov, I. P. (1927). Conditioned reflexes: An investigation of the physiological activity of the cerebral cortex. London: Oxford University Press.

Peña-Correal, T. E., Ordoñez, S., Fonseca, J., Fonseca, L. C. (2012). La investigación empírica de la función sustitutiva referencial. En M. A. Padilla \& R. Pérez-Almonacid (Eds.), La función sustitutiva referencial: Análisis histórico crítico (pp. 35-100). New Orlans, LA: University Press of the South.

Ramírez, N., \& Peña-Correal, T. E. (2011). Historia Funcional de Morfologías Lingüísticas y su Efecto en un Ajuste Convencional. Revista Colombiana de Psicología, 20, 291-305.

Ribes, E. (1997). Causality and contingency: Some conceptual considerations. The Psychological Record, 47, 619-639.

Ribes, E. (1998). La investigación básica concebida como programa científico. En V. Alcaráz \& A. Bouzas (Eds.), Las aportaciones mexicanas a la psicología. La perspectiva de la investigación (pp. 89-101). México: UNAM.

Ribes, E. (2012). Las funciones sustitutivas de contingencias. En M. A. Padilla \& R. PérezAlmonacid (Eds.), La función sustitutiva referencial: Análisis histórico crítico (pp. 19-34). New Orlans, LA: University Press of the South. Ribes, E., Ibáñez, C., \& Hernández-Pozo, M. R. (1986) Hacia una psicología comparativa: Algunas consideraciones conceptuales y metodológicas. Revista Latinoamericana de Psicología, 18, 263-276.

Ribes, E., \& López, F. (1985). Teoría de la conducta: un análisis de campo y paramétrico. México: Trillas.

Ribes, E., Vargas, I., Luna, D., \& Martínez, C. (2009). Adquisición y transferencia de una discriminación condicional en una secuencia 
de cinco criterios distintos de ajuste funcional. Acta Comportamentalia, 17, 129-154.

Sidman, M., \& Tailby, W. (1982). Conditional discrimination vs. matching to sample: an expansion of the testing paradigm. Journal of the Experimental Analysis of Behavior, 37(1), 5-22. doi:10.1901/jeab.1982.37-5

Tonneau, F., \& González, C. (2004). Function transfer in human operant experiments: The role of stimulus pairings. Journal of the Experimental Analysis of Behavior, 81, 239-255. doi:10.1901/jeab.2004.81-239

Tena, O., Hickman, H., Moreno, D., Cepeda, M., \& Larios, R. (2001). Estudios sobre comportamiento complejo. En G. Mares \& Y. Guevara (Eds.), Psicología interconductual: Avances en la investigación básica (pp. 59110). México: Universidad Nacional Autónoma de México-Facultad de Estudios Superiores Iztacala.

Varela, J., \& Quintana, C. (1995). Comportamiento inteligente y su transferencia. Revista Mexicana de Análisis de la Conducta, 21, 47-66.

Auto-referencias de autor: 0

Auto-referencias de la revista: 0 